\title{
Smoked cannabis for spasticity in multiple sclerosis: a randomized, placebo-controlled trial
}

\author{
Jody Corey-Bloom MD PhD, Tanya Wolfson MA, Anthony Gamst PhD, Shelia Jin MD MPH, \\ Thomas D. Marcotte PhD, Heather Bentley BA, Ben Gouaux BA
}

\begin{abstract}
Background: Spasticity is a common and poorly controlled symptom of multiple sclerosis. Our objective was to determine the short-term effect of smoked cannabis on this symptom.
\end{abstract}

Methods: We conducted a placebo-controlled, crossover trial involving adult patients with multiple sclerosis and spasticity. We recruited participants from a regional clinic or by referral from specialists. We randomly assigned participants to either the intervention (smoked cannabis, once daily for three days) or control (identical placebo cigarettes, once daily for three days). Each participant was assessed daily before and after treatment. After a washout interval of 11 days, participants crossed over to the opposite group. Our primary outcome was change in spasticity as measured by patient score on the modified Ashworth scale. Our secondary outcomes included patients' perception of pain (as measured using a visual analogue scale), a timed walk and changes in cognitive function (as measured by patient performance on the
Paced Auditory Serial Addition Test), in addition to ratings of fatigue.

Results: Thirty-seven participants were randomized at the start of the study, 30 of whom completed the trial. Treatment with smoked cannabis resulted in a reduction in patient scores on the modified Ashworth scale by an average of 2.74 points more than placebo ( $p<$ $0.0001)$. In addition, treatment reduced pain scores on a visual analogue scale by an average of 5.28 points more than placebo $(p=0.008)$. Scores for the timed walk did not differ significantly between treatment and placebo $(p=0.2)$. Scores on the Paced Auditory Serial Addition Test decreased by 8.67 points more with treatment than with placebo $(p=0.003)$. No serious adverse events occurred during the trial.

Interpretation: Smoked cannabis was superior to placebo in symptom and pain reduction in participants with treatment-resistant spasticity. Future studies should examine whether different doses can result in similar beneficial effects with less cognitive impact.
$\mathrm{S}$ pasticity is a common and disabling symptom that remains a substantial problem for many patients with multiple sclerosis. Some patients have adverse effects from conventional antispasticity medications; for others, spasticity persists despite treatment. A report from the Institute of Medicine in the United States concluded that the active compounds of cannabis (marijuana) are potentially effective in treating neurologic conditions and "should be tested rigorously in clinical trials." There is evidence that the cannabinoid receptors $\mathrm{CB}_{1}$ and $\mathrm{CB}_{2}$ may be involved in the control of spasticity in multiple sclerosis ${ }^{2}$ and that the endogenous ligand of $\mathrm{CB}_{1}$, anandamide, is itself an effective antispasticity agent. ${ }^{3} \mathrm{CB}_{1}$ receptors are primarily presynaptic; their activation inhibits calcium influx and glutamate release, and reduces neuronal excitability by activating somatic and dendritic potassium channels. ${ }^{4}$
Although many patients with multiple sclerosis endorse smoking cannabis as therapy, evidence that it relieves spasticity is largely anecdotal, as most trials focus on orally administered cannabinoids. We sought to assess the safety and efficacy of smoked cannabis versus placebo in patients with multiple sclerosis who have treatmentresistant spasticity.

\section{Methods}

\section{Participants}

We recruited participants from a regional multiple sclerosis clinic and by referral from specialists. Our eligibility criteria were spasticity and at least moderate increase in tone (score $\geq 3$ points on the modified Ashworth scale ${ }^{5}$ at the elbow, hip or knee). Participants were allowed to continue other treatments for spasticity, with the exception of benzodiazepines, if they had been
Competing interests: None declared.

This article has been peer reviewed.

Correspondence to: Dr. Jody Corey-Bloom, jcoreybloom@ucsd.edu

CMAJ 2012. DOI:10.1503 /cmaj.110837 
taking stable doses for three months or longer. Participants could continue disease-modifying therapy (e.g., interferon $\beta$-1a, interferon $\beta$ - 1 , glatiramer) if they had been on a stable regimen for at least six months. We prohibited any changes to medications that were expected to affect spasticity scores during the trial. Participants could be cannabis-naive or cannabisexposed; if the participants had been previously exposed to cannabis, we asked that they refrain from smoking cannabis for one month before screening and during the trial.

We excluded patients with a history of major psychiatric disorder (other than depression) or substance abuse, substantial neurologic disease other than multiple sclerosis (e.g., epilepsy, head trauma) and severe or unstable medical illnesses, known pulmonary disorders (tuberculosis, asthma), patients who used benzodiazepines to control spasticity or high doses of narcotic medications for pain, and women who were pregnant or breastfeeding.

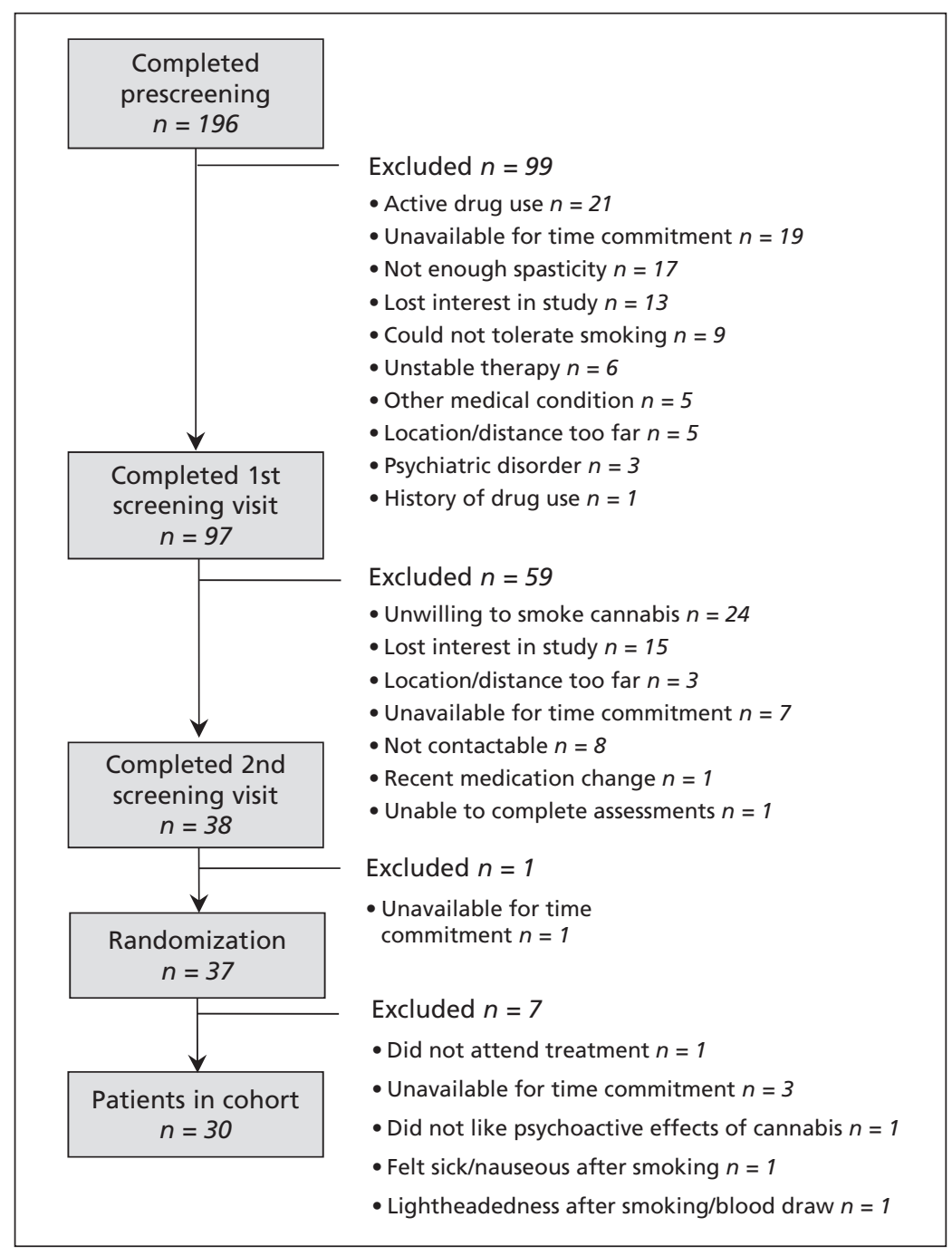

Figure 1: Flow of participants through the study.
Our study was approved by the Human Research Protections Program at the University of California, San Diego, the Research Advisory Panel of California, the Drug Enforcement Administration, the US Food and Drug Administration and the National Institute on Drug Abuse. Our study was monitored by an independent data safety monitoring board through the University of California Center for Medicinal Cannabis Research.

\section{Study design}

We used a randomized, double-blind, placebocontrolled crossover design. We evaluated participants during eight visits over a period of two weeks. Visit 1 was a screening visit during which the participants gave their informed consent. At this time, we took medical/medication histories, screened participants for substance abuse (using urine toxicology) and psychiatric disorders, and determined spasticity using the modified Ashworth scale. ${ }^{5}$ Participants with a positive toxicological screening result (e.g., presence of delta-9 tetrahydrocannabinol, amphetamines, benzodiazepines, cocaine and/or benzoylecgonine) were excluded.

A second screening visit took place within seven days of the first. At this time, we completed the expanded disability status scale, determined spasticity again using the modified Ashworth scale and conducted a battery of cognitive tests to reduce practise effects. During this second visit, participants were given a "practise session" with a placebo cigarette, although they were not told that it was a placebo.

Treatment began within seven days of the second screening visit, including randomization to placebo or smoked cannabis. Phase 1 was followed by an 11-day washout period, after which participants crossed over to the opposite treatment group for phase 2. We assessed each patient before and after treatment for three consecutive days during each phase. The examiner was blind to the treatment group to which each patient was assigned. We assessed patients using the modified Ashworth scale, a visual analog scale for pain, a timed walk and cognitive tests such as the Paced Auditory Serial Addition Test (PASAT). We assessed treatment-emergent effects about 45 minutes after treatment. We collected urine for toxicological screening at the beginning (baseline) of each phase.

We assessed participants at the same time of day to regulate food, medication and time of cannabis intake. Participants smoked either a placebo or a cannabis cigarette, using the Foltin uniform puff procedure (inhalation for $5 \mathrm{~s}$, followed by a 10 -s breath-hold and exhalation, with 
a 45-s wait between puffs), ${ }^{6}$ under supervision in a ventilated room. Participants completed an average of four puffs per cigarette.

Prerolled cannabis and placebo cigarettes with identical appearances and weight (about $800 \mathrm{mg}$ ) were provided by the National Institute on Drug Abuse. Cannabis cigarettes contained about 4\% delta-9-tetrahydrocannabinol (delta-9THC) by weight; placebo cigarettes had the same base material but with the delta-9-THC removed. We chose to use the $4 \%$ delta-9-THC cigarette available from the National Institute on Drug Abuse because it most closely resembled the strength of cigarettes available in the community at the time of the study (typically between $5 \%$ and $6 \%)^{7}$.

We assessed safety and adverse effects by monitoring participants' vital signs in addition to self-report by participants.

\section{Primary outcome}

Our primary outcome was change in spasticity as measured by patient score on the modified Ashworth scale. The modified Ashworth scale ${ }^{5}$ is an ordinal scale ( $0-5$ points) ranking the intensity of muscle tone as follows: 0 , no increase in muscle tone; 1 , slight increase manifested by a catch and release or by minimal resistance at the end of the range of motion when the affected part(s) flexed or extended; 2, slight increase manifested by a catch, followed by minimal resistance throughout the remaining (less than half) range of motion; 3, more marked increase through most of the range of motion, but affected part(s) easily moved; 4, considerable increase in tone, and passive movement is difficult; 5, affected part(s) rigid in flexion and extension. We combined ratings for both elbows, hips and knees for a total possible score of 30 points. We assessed participants using this scale before and about 45 minutes after treatment (cannabis or placebo) at each visit.

This measure has been validated and correlates with motor function. ${ }^{8}$ Although the minimal clinically important difference is not available in the literature, trials using a rating scale of $0-10$ for spasticity have established a threshold of $18 \% .^{9}$ Given this threshold and the mean baseline score of 9 among our participants, a difference of two or more points would be considered clinically meaningful.

\section{Secondary outcomes}

We assessed patients daily for pain (using a visual analogue scale), physical performance (using a timed walk) and cognitive function (the PASAT). We administered these tests before and about 45 minutes after treatment at each visit.

We assessed patients for symptoms using the
Brief Symptom Inventory (BSI), for perceived deficits using the Perceived Deficits Questionnaire (PDQ) and for fatigue using the modified Fatigue Impact Scale (mFIS). We did these assessments before treatment on day 1 and after treatment on day 3 .

In addition, at the end of each visit, we asked

Table 1: Characteristics of patients who completed the protocol

\begin{tabular}{|c|c|}
\hline Characteristic & $\begin{array}{c}\text { Patients, no. (\%)* } \\
n=30\end{array}$ \\
\hline \multicolumn{2}{|l|}{ Sex } \\
\hline Male & $11(37)$ \\
\hline Female & $19(63)$ \\
\hline Age, y, mean (SD) & $51(8)$ \\
\hline Education, y, mean (SD) & $15(2)$ \\
\hline Beck depression inventory, score, mean (SD)† & $9(5)$ \\
\hline \multicolumn{2}{|l|}{ Type of multiple sclerosis } \\
\hline Secondary progressive & $20(67)$ \\
\hline Relapsing-remitting & $10(33)$ \\
\hline Duration of multiple sclerosis, y, mean (SD) & $8.5(7.4)$ \\
\hline Expanded disability status scale, score, mean (SD) $\ddagger$ & $5.3(1.5)$ \\
\hline \multicolumn{2}{|l|}{ Modified Ashworth scale, score, mean (SD)§ } \\
\hline Combined & $9.3(2.3)$ \\
\hline Elbow & $2.5(1.2)$ \\
\hline Hip & $3.3(1.2)$ \\
\hline Knee & $3.6(1.2)$ \\
\hline \multicolumn{2}{|l|}{ Requires mobility aids } \\
\hline Requires any aid & $20(67)$ \\
\hline Requires a cane & $9(30)$ \\
\hline Requires a walker & $5(17)$ \\
\hline Requires a wheelchair & $6(20)$ \\
\hline \multicolumn{2}{|l|}{ Undergoing disease-modifying therapy } \\
\hline Any disease-modifying therapy & $21(70)$ \\
\hline Interferon $\beta-1 \mathrm{a}$ & $9(30)$ \\
\hline Interferon $\beta-1 b$ & $6(20)$ \\
\hline Glatiramer & $6(20)$ \\
\hline \multicolumn{2}{|l|}{ Antispasticity agents } \\
\hline Any antispasticity agent & $18(60)$ \\
\hline Baclofen & $14(47)$ \\
\hline Tizanidine & $4(13)$ \\
\hline \multicolumn{2}{|l|}{ Previous exposure to cannabis } \\
\hline Any exposure & $24(80)$ \\
\hline Exposure in previous year & $10(33)$ \\
\hline$>1$ year since last use & $14(47)$ \\
\hline \multicolumn{2}{|c|}{$\begin{array}{l}\text { Note: SD = standard deviation. } \\
\text { *Unless otherwise indicated. } \\
\text { †Scores range from } 0 \text { to } 63 \text {, with higher scores suggesting a greater severity of depressive } \\
\text { symptoms. } \\
\text { fScores range from } 0 \text { to } 10 \text {, with higher scores suggesting a greater degree of disability. } \\
\text { §As determined during screening visit } 1 \text {. Scores range from } 0 \text { to } 24 \text {, with higher scores } \\
\text { suggesting greater spasticity. }\end{array}$} \\
\hline
\end{tabular}




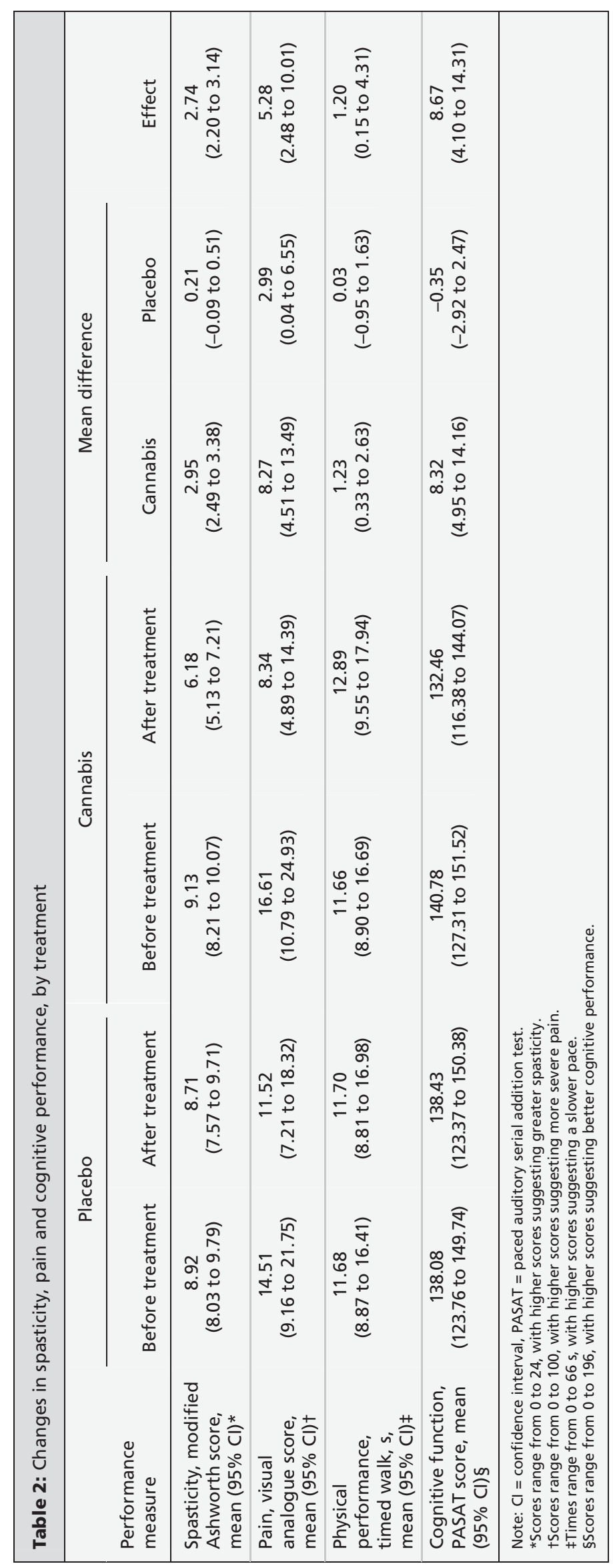

patients to assess their feeling of "highness" after treatment, according to question 1 from the Subjective Ratings of High and Sedation Questionnaire (SRHS-R), and to guess which treatment they were receiving (placebo or cannabis).

Detailed descriptions of these measures are available in Appendix 1 (available at www.cmaj.ca /lookup/suppl/doi:10.1503/cmaj.110837/-/DC1).

\section{Statistical analysis}

We calculated mean scores (and 95\% confidence intervals [CIs]) on the modified Ashworth scale during each visit of each phase, at each assessment time (before and after treatment). We calculated bootstrap-based, biascorrected, accelerated CIs for extra precision around each mean. We calculated four overall mean scores on the modified Ashworth scale (before and after smoking during both phases). We compared the difference in scores before and after smoking for each of the two phases using paired $t$ tests. We then compared the change in this difference (after to before) in the two phases (placebo and active) using a paired $t$ test. We used the same analysis to examine scores on the visual analogue scale for pain, the timed walk and the PASAT.

We analyzed secondary variables according to the schedule of measurements. We calculated the means and bootstrap-based CIs for patient scores on the BSI, the PDQ, and the mFIS for each day on which these measures were assessed (day 1 before smoking, day 3 after smoking). We as sessed the overall differences for before and after treatment with placebo and cannabis using a paired $t$ test. We calculated the means and bootstrapbased CIs for the answer to question 1 of the SRHS-R questionnaire. We used a paired $t$ test to compare the overall difference in "highness" between treatment and placebo.

We performed power calculations before the beginning of the study, and these were reviewed and approved by an external scientific advisory board and regulatory agencies. A priori, we identified as "clinically important" any departure from zero in the hypothesized direction that is greater than one standard deviation (SD) of paired differences. We determined that a sample size of 30 would yield better than $80 \%$ power $(\alpha=0.05)$ to detect such an effect size.

\section{Results}

\section{Study participants}

We identified 196 patients for screening (Figure 1). Of these patients, 38 completed both screening visits, 37 were randomized, and 30 
completed the study. (Seven patients withdrew before completion.)

Of the 30 patients who completed the protocol, 63\% were women (Table 1). The average age of participants was about 50 years, and the average level of education was 15 years in school. The mean score on the expanded disability status scale was 5.3 (SD 1.5). Sixtyseven percent of participants required walking aids, and $20 \%$ required the use of a wheelchair. The score on the modified Ashworth scale at the initial screening visit was a mean of 9.3 (SD 2.3). Seventy percent of participants were undergoing disease-modifying therapy, and $60 \%$ were taking antispasticity agents. Most of the participants $(80 \%)$ had previous recreational experience with cannabis, and $33 \%$ of participants had used cannabis within the previous year.

\section{Primary outcome}

Smoking cannabis reduced patient scores on the modified Ashworth scale by an average of 2.74 points (95\% bootstrap CI 2.20 to 3.14) more than placebo $(p<0.001)$ (Table 2 and Figure 2$)$. The order of treatment (cannabis in phase 1 or phase 2) did not significantly affect the outcome $(p=0.8)$.

\section{Secondary outcomes}

Smoking cannabis reduced patient scores on the visual analogue scale by 5.28 points $(95 \%$ bootstrap CI 2.48 to 10.01) more than placebo ( $p=$ 0.008 ) (Table 2). The difference between timed walk scores in the two conditions was not significant $(p=0.2)$.

Participants in both conditions showed improvement on the PASAT over the three visits, consistent with practise effects. However, within sessions, the group smoking cannabis had a consistent reduction in performance after the drug was administered versus before. Overall, smoking cannabis reduced scores on this test by 8.67 points ( $95 \%$ bootstrap CI 4.10 to 14.31) more than placebo $(p=0.003)$ (Table 2).

Smoking cannabis did not significantly affect patient perceptions of fatigue or deficits, nor did it increase symptoms, but it did increase patient perception of "highness" by 5.04 points more than placebo $(p<0.001)$ (Table 3$)$. Seventeen participants correctly guessed their treatment phase for all six visits, and one participant guessed cannabis for all days (data not shown). For the remaining participants, cannabis was correctly guessed on $33 / 35$ visits; they correctly guessed placebo on 21/36 visits (data not shown).
A

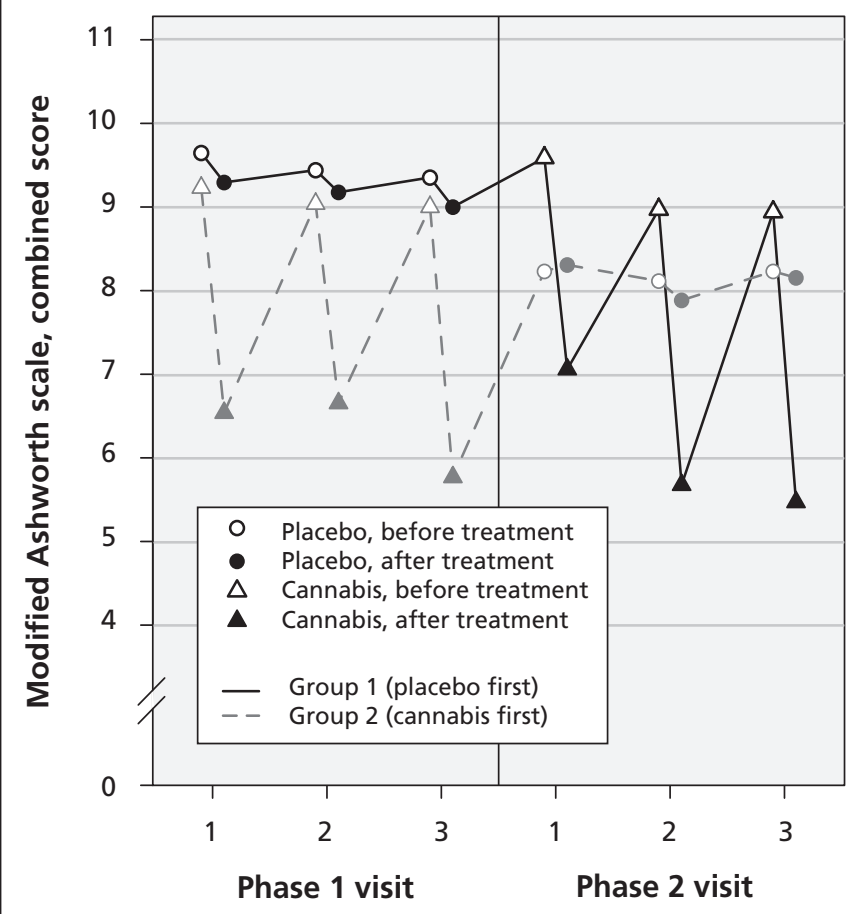

B

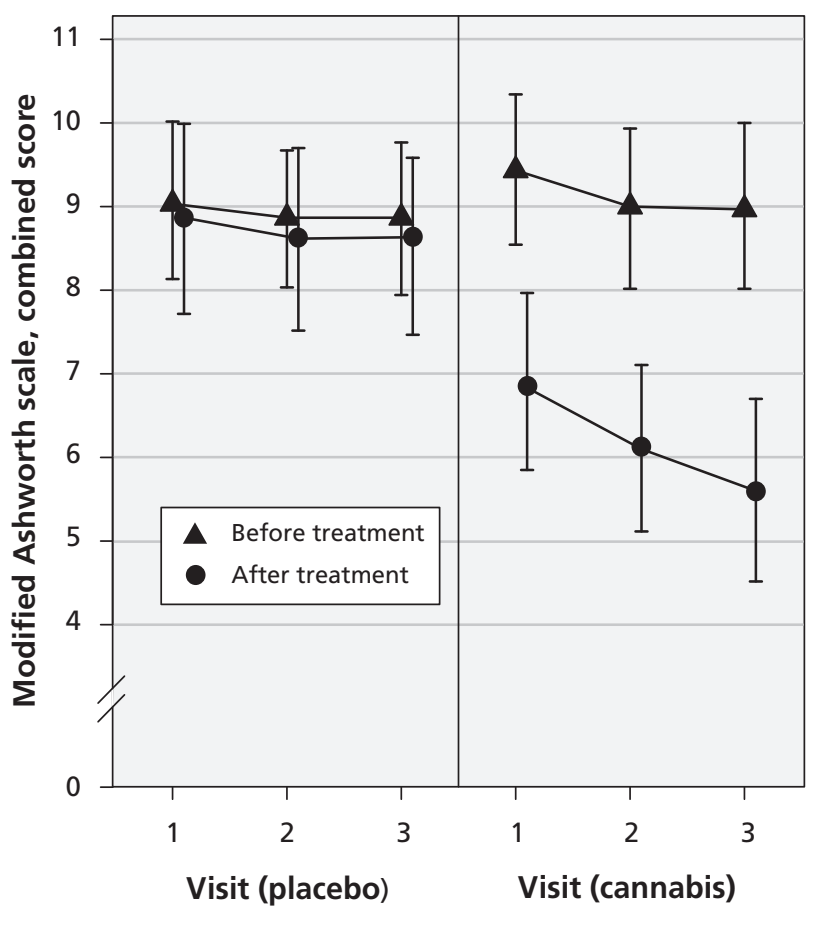

Figure 2: Spasticity as measured by mean combined scores on the modified Ashworth scale, before and after treatment, on each day of each phase of the trial. (A) Change in scores by phase, before and after crossover. (B) Change in scores before and after treatment with placebo versus cannabis. 


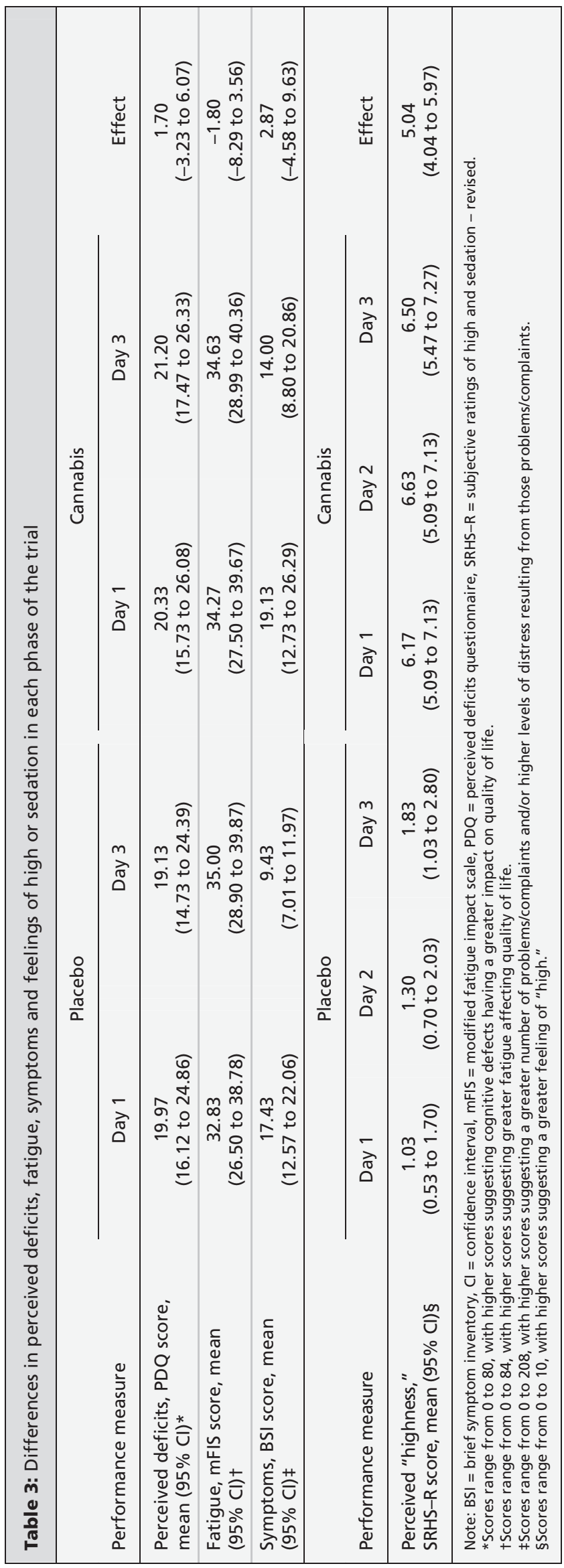

\section{Sensitivity analyses}

Using a worst-case scenario sensitivity analysis (assuming that the seven patients who withdrew would not have shown any treatment effect), compared with placebo, smoking cannabis reduced average scores on the modified Ashworth scale by 2.22 points $(p<0.001)$, on the visual analogue scale of pain by 4.28 points $(p=0.009)$ and on the PASAT by 6.981 points $(p=0.003)$. These findings suggest that dropouts had no meaningful effect on our results.

\section{Safety effects}

Although smoked cannabis was generally welltolerated, patients reported more adverse effects during the active phase than during the placebo phase (Table 4). Withdrawals from treatment were due to adverse events (two patients felt uncomfortably "high", two had dizziness and one had fatigue), the schedule being too demanding (one patient) and pain unrelated to the study (one patient). Of the patients who withdrew, three had no previous exposure to cannabis, two had only some exposure ( $>1 \mathrm{yr}$ since last use) and two had been exposed during the previous year. None of our participants had episodes of hypertension, hypotension, tachycardia or bradycardia requiring medical intervention.

\section{Interpretation}

\section{Main findings}

We saw a beneficial effect of smoked cannabis on treatment-resistant spasticity and pain associated with multiple sclerosis among our participants. Although generally well-tolerated by our participants, smoking cannabis was accompanied by acute cognitive effects.

\section{Comparison with other studies}

Other studies have investigated the effect of orally administered cannabinoids on spasticity related to multiple sclerosis, including oromucosal sprays and capsules containing cannabis extract, with mixed results. Any reductions in spasticity have generally only been seen on subjective ratings. ${ }^{10,11}$ One study showed a reduction of 1.82 points on the original Ashworth spasticity scale (baseline mean 22) compared with a reduction of 0.23 points for placebo. ${ }^{12}$ In a trial of a cannabis-based oromucosal spray, Collin and colleagues ${ }^{13}$ found that $40 \%$ of patients selfreported a benefit of $30 \%$ or more over a period of six weeks using a numerical rating scale of spasticity. Changes in the scores on the Ashworth spasticity scale did not significantly differ between participants receiving treatment and 
those getting the placebo. Similar results were found in a study involving 337 patients with treatment-resistant spasticity. ${ }^{14}$

With respect to commonly used medications, sublingual tizanidine hydrochloride resulted in a 3.0-point decline in Ashworth scores when evaluated after one week of treatment, in comparison with a 1.81-point decline with oral tizanidine and a 1.19-point decline with placebo. ${ }^{15}$ Baseline mean Ashworth scores for the patients in this study had ranged from 8.31 to 11.31 . In addition, a study involving patients who were not responsive to oral baclofen found that intrathecal delivery resulted in a change in mean Ashworth scores from 4.0 to $1.2 .^{16}$

We saw significant reduction in the pain felt by our participants. Although orally administered cannabinoids failed to improve pain in an uncontrolled study involving 20 patients with multiple sclerosis, ${ }^{17}$ two placebo-controlled studies $^{10,18}$ did find a treatment effect. In a trial of a sublingual spray containing delta-9-THC alone or combined with cannabinol, Rog and colleagues reported a $41 \%$ reduction in pain, compared with a $22 \%$ reduction with placebo. ${ }^{18}$ Literature on cannabinoids for pain conditions other than multiple sclerosis is limited, although three recent randomized placebo-controlled trials of smoked cannabis found significant reductions in neuropathic pain. ${ }^{19-21}$ Our participants began with relatively low levels of pain; future studies might focus on patients with more intense pain.

Previous studies that have used different delivery systems (pills, oral mucosal sprays) and evaluated participants at study completion rather than within one hour of smoking have reported no or limited adverse effects on cognition. . $^{11,18,22,23}$ However, smoked cannabis was associated with acute cognitive effects among the participants of our study, as shown by their performances on the PASAT. The clinical significance of this result is uncertain; despite the transient decrease in scores, patients were still within normal ranges for their ages and levels of education. ${ }^{24}$ It is worth noting that conventional treatments such as baclofen and tizanidine hydrochloride may also affect cognition, ${ }^{25}$ although published data are scarce. Recently, concerns have been cited regarding the potential long-term cognitive effects of cannabis use in patients with multiple sclerosis. ${ }^{26,27}$ Although these studies have had small samples and other limitations, ${ }^{28,29}$ the issue warrants further attention.

\section{Limitations}

Many participants had previously used cannabis, raising the possibility of self-selection bias based
Table 4: Prevalence of adverse effects during the trial, by group

\begin{tabular}{|c|c|c|}
\hline \multirow[b]{2}{*}{ Adverse effect } & \multicolumn{2}{|c|}{ Treatment group, no. (\%) } \\
\hline & Cannabis & Placebo \\
\hline Dizziness & $8(23)$ & $1(3)$ \\
\hline Headache & $7(20)$ & $6(19)$ \\
\hline Fatigue & $7(20)$ & $2(6)$ \\
\hline Nausea & $4(11)$ & 1 (3) \\
\hline Feeling "too high" & $2(6)$ & $0 \quad(0)$ \\
\hline Throat irritation & 1 (3) & 1 (3) \\
\hline
\end{tabular}

on their previous positive response to the drug. Thus, our results might not be generalizable to patients who are cannabis-naïve.

It is difficult to completely blind participants to psychoactive substances. Participants could generally tell which treatment they were receiving, although this is unlikely to affect objectively assessed spasticity scores. Although the literature on the reliability of the modified Ashworth scale shows mixed opinion, we selected this measure because it is the most widely used in clinical practice, and any measurement limitations would likely result in increased variance and a failure to find a treatment effect.

\section{Conclusion}

Using an objective measure, we saw a beneficial effect of inhaled cannabis on spasticity among patients receiving insufficient relief from traditional treatments. Although generally welltolerated, smoking cannabis had acute cognitive effects. Larger, long-term studies are needed to confirm our findings and determine whether lower doses can result in beneficial effects with less cognitive impact.

\section{References}

1. Watson SJ, Benson JA Jr, Joy JE. Marijuana and medicine: assessing the science base: a summary of the 1999 Institute of Medicine report. Arch Gen Psychiatry 2000;57:547-52.

2. Pryce G, Baker D. Control of spasticity in a multiple sclerosis model is mediated by $\mathrm{CB} 1$, not $\mathrm{CB} 2$, cannabinoid receptors. $\mathrm{Br}$ J Pharmacol 2007;150:519-25.

3. Brooks JW, Pryce G, Bisogno T, et al. Arvanil-induced inhibition of spasticity and persistent pain: evidence for therapeutic sites of action different from the vanilloid VR1 receptor and cannabinoid $\mathrm{CB}(1) / \mathrm{CB}(2)$ receptors. Eur J Pharmacol 2002; 439:83-92.

4. Smith PF. New approaches in the management of spasticity in multiple sclerosis patients: role of cannabinoids. Ther Clin Risk Manag 2010;6:59-63.

5. Bohannon RW, Smith MB. Interrater reliability of a modified Ashworth scale of muscle spasticity. Phys Ther 1987;67:206-7.

6. Foltin RW, Ficshman MW, Nellis MJ, et al. Marijuana effects and behavioral contingencies. NIDA Res Monogr 1986;67: 355-61.

7. Mehmedic Z, Chandra S, Slade D, et al. Potency trends of Delta9-THC and other cannabinoids in confiscated cannabis preparations from 1993 to 2008. J Forensic Sci 2010;55:1209-17.

8. Katz RT, Rovai GP, Brait C, et al. Objective quantification of 
spastic hypertonia: correlation with clinical findings. Arch Phys Med Rehabil 1992;73:339-47.

9. Farrar JT, Troxel AB, Stott C, et al. Validity, reliability, and clinical importance of change in a 0-10 numeric rating scale measure of spasticity: a post hoc analysis of a randomized, double-blind, placebo-controlled trial. Clin Ther 2008;30:974-85.

10. Zajicek J, Fox P, Sanders H, et al. Cannabinoids for treatment of spasticity and other symptoms related to multiple sclerosis (CAMS study): multicentre randomised placebo-controlled trial. Lancet 2003;362:1517-26.

11. Wade DT, Makela P, Robson P, et al. Do cannabis-based medicinal extracts have general or specific effects on symptoms in multiple sclerosis? A double-blind, randomized, placebo-controlled study on 160 patients. Mult Scler 2004;10:434-41.

12. Zajicek JP, Sanders HP, Wright DE, et al. Cannabinoids in multiple sclerosis (CAMS) study: safety and efficacy data for 12 months follow up. J Neurol Neurosurg Psychiatry 2005;76:1664-9.

13. Collin C, Davies P, Mutiboko IK, et al. Randomized controlled trial of cannabis-based medicine in spasticity caused by multiple sclerosis. Eur J Neurol 2007;14:290-6.

14. Collin C, Ehler E, Waberzinek G, et al. A double-blind, randomized, placebo-controlled, parallel-group study of Sativex, in subjects with symptoms of spasticity due to multiple sclerosis. $\mathrm{Neu}$ rol Res 2010;32:451-9.

15. Vakhapova V, Auriel E, Karni A. Nightly sublingual tizanidine $\mathrm{HCl}$ in multiple sclerosis: clinical efficacy and safety. Clin $\mathrm{Neu}$ ropharmacol 2010;33:151-4.

16. Penn RD, Savoy SM, Corcos D, et al. Intrathecal baclofen for severe spinal spasticity. N Engl J Med 1989;320:1517-21.

17. Centonze D, Mori F, Koch G, et al. Lack of effect of cannabisbased treatment on clinical and laboratory measures in multiple sclerosis. Neurol Sci 2009;30:531-4.

18. Rog DJ, Nurmikko TJ, Friede T, et al. Randomized, controlled trial of cannabis-based medicine in central pain in multiple sclerosis. Neurology 2005;65:812-9.

19. Abrams DI, Jay CA, Shade SB, et al. Cannabis in painful HIVassociated sensory neuropathy: a randomized placebo-controlled trial. Neurology 2007;68:515-21.

20. Ellis RJ, Toperoff W, Vaida F, et al. Smoked medicinal cannabis for neuropathic pain in HIV: a randomized, crossover clinical trial. Neuropsychopharmacology 2009;34:672-80.

21. Ware MA, Wang T, Shapiro S, et al. Smoked cannabis for chronic neuropathic pain: a randomized controlled trial. CMAJ 2010;182:E694-701.

22. Vaney C, Heinzel-Gutenbrunner M, Jobin P, et al. Efficacy, safety and tolerability of an orally administered cannabis extract in the treatment of spasticity in patients with multiple sclerosis: a randomized, double-blind, placebo-controlled, crossover study. Mult Scler 2004;10:417-24.

23. Aragona M, Onesti E, Tomassini V, et al. Psychopathologica and cognitive effects of therapeutic cannabinoids in multiple sclerosis: a double-blind, placebo controlled, crossover study. Clin Neuropharmacol 2009;32:41-7.

24. Heaton RK, et al. Revised comprehensive norms for an expanded Halstead-Reitan battery: demographically adjusted neuropsychological norms for African American and Caucasian adults. Lutz (FL): Psychological Assessment Resources; 2004.

25. Shah J, Wesnes KA, Kovelesky RA, et al. Effects of food on the single-dose pharmacokinetics/pharmacodynamics of tizanidine capsules and tablets in healthy volunteers. Clin Ther 2006;28:1308-17.

26. Ghaffar O, Feinstein A. Multiple sclerosis and cannabis: a cognitive and psychiatric study. Neurology 2008;71:164-9.

27. Honarmand K, Tierney MC, O'Connor P, et al. Effects of cannabis on cognitive function in patients with multiple sclerosis. Neurology 2011;76:1153-60.

28. Arnett PA. Cannabis bliss? Perhaps not? Neurology 2008;71:160-1.

29. Messinis L, Papathanasopoulos P. Multiple sclerosis and cannabis: a cognitive and psychiatric study. Neurology 2009;72: 100-1, author reply 101 .

Affiliations: From the Departments of Neurosciences (Corey-Bloom), Biostatistics (Wolfson, Gamst, Jin) and Psychiatry (Marcotte, Bentley); and the Center for Medicinal Cannabis Research (Marcotte, Bentley, Gouaux), University of California, San Diego, La Jolla, Calif.

Contributors: Jody Corey-Bloom oversaw the design and implementation of the study design, and the analysis and interpretation of the data, and drafted the manuscript. Tanya Wolfson, Anthony Gamst and Shelia Jin participated in the designing the study design and conducted the statistical analysis. Thomas D. Marcotte participated in drafting the manuscript and interpreted the results of the neuropsychological tests. Heather Bentley and Ben Gouaux assisted with the design and implementation of the study and helped draft the manuscript. All of the authors approved the final version of the manuscript submitted for publication.

Funding: This study was funded by grant number C00-SD-103 from the University of California, Center for Medicinal Cannabis Research (CMCR). The CMCR assisted the investigators in obtaining the State and Federal approvals for this project.

For the study protocol pertaining to this article, please see Appendix 2, available at www.cmaj.ca /lookup/suppl/doi:10.1503/cmaj.110837/-/DC1. 